\title{
Synthesis and Microanalysis of Aligned Carbon Nanotube Arrays
}

\author{
Devon McClain*, Lifeng Dong*, Chiaching Pan*, Jun Jiao*, Coralee McCarter**, David \\ Bahr**, Cecilia Richards**, Robert Richards** \\ *Physics Department, Portland State University, Portland OR 97207 \\ **School of Mechanical and Materials Eng., Washington State Univ. Pullman WA 99164
}

Carbon nanotubes (CNTs) have been considered promising candidates for thermal devices due to their high thermal conductivity. Indeed, the multi-walled carbon nanotube (MWCNT) has demonstrated a thermal conductivity of more than $3000 \mathrm{~W} / \mathrm{mK}$ at room temperature [1]. However, randomly aligned MWCNT films have displayed much lower thermal conductivities in the range of $25-35 \mathrm{~W} / \mathrm{mK}$ [2]. This suggests that it is possible to exploit the much higher thermal conductivity of CNTs by using well-aligned arrays capable of improved thermal coupling.

CNT growth was accomplished via chemical vapor deposition (CVD) method. The substrate was prepared as follows. A silicon wafer (100) was etched for 30 seconds in Buffered Oxide Etch (BOE). An iron nitrate $(1.5 \mathrm{M}, 15 \mathrm{~mL})$ sol gel catalyst was spun onto the wafer at 3000 rpm for 30 seconds and dried. Photoresist was spun on at $3000 \mathrm{rpm}$ for 30 seconds and then patterned with a 1600 dot array using an exposure time of 12 seconds and an intensity of 10 $\mathrm{mW} / \mathrm{cm}^{2}$. The wafer was diced and the individual dies subjected to various BOE etch times. The sol gel patterned die were placed in a horizontal tube furnace for MWCNT growth. Growth was achieved at $700^{\circ} \mathrm{C}$ for 30 minutes with an admixture of $\mathrm{H}_{2}$ and $\mathrm{C}_{2} \mathrm{H}_{2}(385 \mathrm{sccm}$ and $25 \mathrm{sccm}$ respectively) as in Dong et al [3].

Characterization was performed using a field emission scanning electron microscope (FEI Sirion FESEM) and a high-resolution transmission electron microscope (Tecnai F-20 HRTEM) equipped with an energy dispersive x-ray spectrometer (EDS). Fig. 1(a) depicts a typical sol gel catalyst dot after patterning on the substrate. Dots are roughly $1 \mu \mathrm{m}$ in height, $15-20 \mu \mathrm{m}$ in diameter and spaced every $100 \mu \mathrm{m}$. Fig. 1(b) is an overview of one section of the post-growth array of CNTs. Note that the CNTs are well-defined and restricted to catalyst dot locations. Fig. 2(a) is a low magnification SEM image of a CNT column. The inset is of one section of the column's sidewall showing the vertically aligned CNTs at high magnification. A TEM image [Fig. 2(b)] of a bundle of CNTs from the same specimen indicates that most of CNTs are $5-10 \mathrm{~nm}$ in diameter with lengths in excess of $20 \mu \mathrm{m}$. Fig. 3(a) is an HRTEM image revealing that the CNT consists of 5-6 graphitic layers. The surface of the tube is partially covered by amorphous carbon. The low number of layers present in these MWCNTs also suggests the potential of this method for producing double-walled or single-walled nanotubes. The EDS spectrum in Fig. 3(b) confirms that iron catalyst leads the growth of the CNTs. The signals of $\mathrm{Si}, \mathrm{O}$, and $\mathrm{Cu}$ in the spectrum are generated from the substrate and TEM grid. The inset of Fig. 3(b) shows the presence of Fe catalyst within the tip of a MWCNT. The EDS signals were generated from the location of the target. Further study is underway to characterize the thermal conductivity of this structure. 


\section{References}

[1] P. Kim, L. Shi, A. Majumdar, and P. L. McEuen, Phys. Rev. Lett. 87, 215502 (2001).

[2] D. Yang et al, Phys. Rev. B, 66, 165440 (2002).

[3] L. Dong, J. Jiao, C. Pan, D. Tuggle, Appl. Phys. A, 78, 9-14 (2004).

Acknowledgments: Financial support for this research was provided in part by the NSF under awards No. CTS-040370, DMR-035738 (REU Site) and by the US Army SMDC under contract No. DASG60-02-C0001.

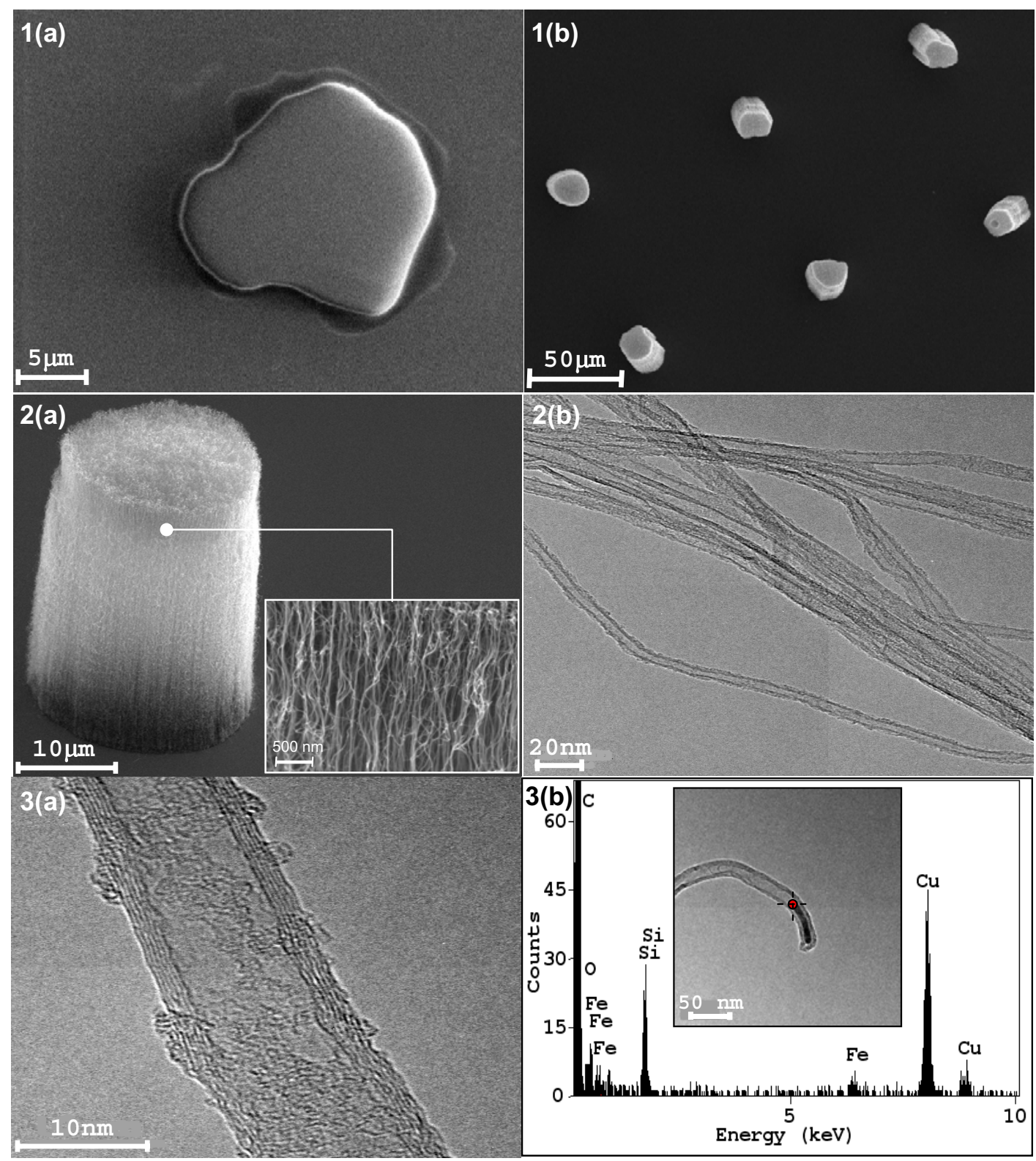

Fig. 1(a) shows a single pre-growth ferrous nitrate catalyst dot while Fig. 1(b) reveals well confined CNT growth at catalyst dot locations.

Fig. 2(a) shows a vertically aligned CNT column rising from the location of a catalyst dot.

Fig. 2(b) is a TEM micrograph of a CNT bundle revealing CNT diameters of 5-10nm.

Fig. 3(a) is a HRTEM showing a MWCNT with the surface partially covered by some amorphous carbon. Fig. 3(b) is an EDS spectrum revealing the Fe catalyst inside the CNTs. 